\title{
Philosophiques
}

\section{Un problème de la légitimité politique dans la pensée de}

\section{Rousseau}

\section{Norbert Lenoir}

Volume 27, numéro 2, automne 2000

URI : https://id.erudit.org/iderudit/004945ar

DOI : https://doi.org/10.7202/004945ar

Aller au sommaire du numéro

\section{Éditeur(s)}

Société de philosophie du Québec

\section{ISSN}

0316-2923 (imprimé)

1492-1391 (numérique)

Découvrir la revue

\section{Citer cet article}

Lenoir, N. (2000). Un problème de la légitimité politique dans la pensée de Rousseau. Philosophiques, 27(2), 323-350. https://doi.org/10.7202/004945ar

\section{Résumé de l'article}

Dans le Contrat Social, Rousseau semble fonder la légitimité politique sur deux institutions : le Souverain, siège de la puissance législative et le Gouvernement, lieu de la puissance exécutive. Pourtant, dans le livre IV du même ouvrage, il définit la nécessité d'une troisième institution : le Tribunat, situé en tiers entre le Souverain et le Gouvernement. Pour quelle raison Rousseau détermine-t-il la nécessité de ce troisième pouvoir pour fonder la légitimité démocratique ? La raison provient de la dynamique initiée par le Gouvernement qui, tout en étant une institution légitime et nécessaire, tend à s'approprier le pouvoir législatif. Rousseau développe une pensée politique qui prend conscience de la fragilité de la légitimité démocratique, fragilité qui peut se définir par sa tendance à créer, au travers de ses institutions, des volontés indépendantes des citoyens qui défendent des intérêts particuliers en prenant le masque de la volonté générale. Face à ce problème, Rousseau caractérise trois moyens politiques, afin de donner une consistance à la légitimité démocratique : le "Tribunat ", "le Droit de Représentation " et enfin la circulation du pouvoir. 


\title{
Un problème de la légitimité politique dans la pensée de Rousseau
}

\author{
NORBERT LENOIR
}

RÉSUMÉ. - Dans le Contrat Social, Rousseau semble fonder la légitimité politique sur deux institutions : le Souverain, siège de la puissance législative et le Gouvernement, lieu de la puissance exécutive. Pourtant, dans le livre IV du même ouvrage, il définit la nécessité d'une troisième institution : le Tribunat, situé en tiers entre le Souverain et le Gouvernement. Pour quelle raison Rousseau détermine-t-il la nécessité de ce troisième pouvoir pour fonder la légitimité démocratique ? La raison provient de la dynamique initiée par le Gouvernement qui, tout en étant une institution légitime et nécessaire, tend à s'approprier le pouvoir législatif. Rousseau développe une pensée politique qui prend conscience de la fragilité de la légitimité démocratique, fragilité qui peut se définir par sa tendance à créer, au travers de ses institutions, des volontés indépendantes des citoyens qui défendent des intérêts particuliers en prenant le masque de la volonté générale. Face à ce problème, Rousseau caractérise trois moyens politiques, afin de donner une consistance à la légitimité démocratique : le «Tribunat», «le Droit de Représentation » et enfin la circulation du pouvoir.

ABSTRACT. - In the Contrat Social, Rousseau seems to base political legitimacy on two institutions, the sovereign power, seat of legislative power and the government, place of executive power. Nevertheless, in the fourth book of the same work, he defines the necessity of a third political institution, the "Tribunat", situated entirely between the sovereign power and the government. For what reason does Rousseau determine the necessity of this third power as the base of democratic legitimacy? The reason is the result of the dynamics initiated by the government who, while at the same time being a legitimate and necessary power, tend to take over the legislative power. Rousseau develops a political thinking which realises the fragility of democratic legitimacy, fragility which can be described by its tendency to create, through these institutions, citizen own independent wishes who are defending particular interests in taking the façade of the general will. Facing this problem, Rousseau characterises three political measures in order to give substance to the democratic legitimacy: the "Tribunat", the "Droit de Représentation", and finally the circulation of the power.

Rousseau, en donnant au Contrat Social le sous-titre Principes du Droit Politique, souhaite inscrire cet ouvrage dans une interrogation sur la légitimité politique. Mais, on a souvent tendance à bloquer cette analyse autour des notions de souveraineté et de volonté générale ${ }^{1}$. Ces deux notions ne sauraient à elles seules donner au Contrat Social tous les éléments constitutifs de sa légitimité politique. Et cela pour deux raisons.

1. Nous ne voulons surtout pas affirmer que toutes les interprétations de la pensée politique de Rousseau se contentent de définir les seules notions de souveraineté et de volonté

PHILOSOPHIQUES 27/2 - Automne 2000, p. 323-350 
Premièrement, la légitimité du politique ne peut pas se réduire à une détermination du souverain et de sa volonté spécifique, puisque Rousseau fait intervenir en complément le Législateur qui représente la figure symbolique du savoir. ${ }^{2}$

Deuxièmement, Rousseau interroge la légitimité politique dans les termes du pouvoir avec la nécessité du Gouvernement. Le Gouvernement est défini comme puissance exécutive de la volonté générale. La légitimité du politique, dans le Contrat Social ne peut pas uniquement s'interroger dans les termes de la volonté, car elle doit intégrer l'institutionnalisation de la force, seule capable de donner affectivité à la loi : " la perfection de l'ordre social consiste, il est vrai, dans le concours de la force et de la loi ; mais il faut pour cela que la loi dirige la force ${ }^{3}$. C'est ce rapport nécessaire entre la

générale. Ce serait oublier les belles études de Robert Derathé sur le gouvernement dans son ouvrage J.J. Rousseau et la science politique de son temps, Paris, Vrin, 1979 et dans son article, paru dans les Annales de philosophie politique $N^{\circ} 5$ en 1965, "Les rapports de l'exécutif et du législatif chez J.J. Rousseau ». On peut aussi mentionner, toujours au sujet du gouvernement, l'article de B. de Jouvenel : "Théorie des formes de Gouvernement chez Rousseau » dans Commentaires du Contrat Social - Pluriel - Hachette 1978. Relativement à la figure du Législateur dans l'œuvre de Rousseau, on peut mentionner l'article de B. Gagnebin : "Le rôle du législateur dans les conceptions politiques de Rousseau ", dans Études sur le Contrat Social, Paris, les belles Lettres, 1964. Il ne faudrait pas omettre l'ouvrage d'Alfred Cobban, Rousseau and the modern State, Second revised Edition, Londres, George Allen \& Unwin, 1964. Nous voulons réfléchir sur la légitimité politique à partir du principe de la subordination de l'exécutif au législatif, car il pose un problème que le philosophe genevois tente de résoudre à l'aide d'institutions telles que le "Tribunat » ou le " droit de représentation » qui ont, peut-être, été laissées dans l'ombre par les interprètes de Rousseau. Nous ne prétendons pas que cette question soit la seule question de la légitimité du politique chez J.J. Rousseau, c'est pour cette raison que notre titre est Un problème de la légitimité politique dans la pensée de J.J. Rousseau et non pas Le problème de la légitimité politique dans la pensée de J.J. Rousseau. Ce problème possède, pour nous, une valeur interprétative, car il peut présenter une cohérence entre le Contrat Social, Les Lettres écrites de la montagne, surtout les Lettres 8 et 9, et certains passages des Considérations sur le Gouvernement de Pologne, non pas sous un jour radicalement nouveau, mais dans une perspective différente.

2. C'est bien en terme de connaissance que Rousseau en appelle à la nécessité du Législateur : "De lui-même le peuple veut toujours le bien, mais de lui-même il ne le voit pas toujours [...]. Voilà d'où naît la nécessité d'un Législateur ». (Contrat Social, II, 6, Cuvres Complètes de la Pléiade, t. III, p 380). La volonté souveraine, qui s'identifie à celle du peuple pour Rousseau, trouve dans le Législateur son complément, celui d'une volonté politique toujours éclairée par la connaissance d'un bien politique jamais "séduit par des volontés particulières » (Ibid). Le Législateur représente la symbolique d'une volonté qui coïnciderait avec une connaissance transparente du politique, qui aurait pour condition de possibilité le détachement absolu de tout intérêt particulier. Cette symbolique donne sens à l'action du souverain. Elle lui signale que tout acte législatif tente de réaliser cette coïncidence entre la volonté politique et l'absence d'intérêt particulier. Certes, cette coïncidence parfaite est impossible à réaliser, sûrement réservée "à un peuple de Dieux » (Ibid. III, 3, p. 406), mais elle représente l'horizon de toute législation légitime.

3. Écrits sur l'abbé de Saint-Pierre, L'état de guerre, distinctions fondamentales, t. III, p. 606. 
volonté du Souverain et la puissance du Gouvernement qui crée un problème au sein du Contrat Social.

Le Contrat Social, en déterminant une légitimité démocratique, fondée sur une théorie de la volonté générale, installe un problème au cœur même de cette légitimité. Ce problème apparaît en mettant en parallèle deux textes du Contrat Social.

Dans le premier, Rousseau affirme :

Toute action libre a deux causes qui concourent à la produire, l'une morale, savoir la volonté qui détermine l'acte, l'autre physique, savoir la puissance qui l'exécute [...]. Le corps politique a les mêmes mobiles ; on y distingue de même la force et la volonté ; celle-ci sous le nom de puissance législative, l'autre sous le nom de puissance exécutive. Rien ne s'y fait ou ne doit s'y faire sans leur concours ${ }^{4}$.

Rousseau affirme ici que la légitimité politique est fondée par deux institutions, la puissance législative et la puissance exécutive, qu'il identifie respectivement au Souverain et au Gouvernement.

La puissance législative "appartient au peuple, et ne peut appartenir qu'à lui $»^{5}$. Elle n'est que l'exercice de la volonté générale et n'agit que par "des lois et ne saurait agir autrement ${ }^{6}$.

La deuxième institution nécessaire est la puissance exécutive qui donne affectivité aux lois. Rousseau caractérise la fonction du Gouvernement par la "réduction des lois en actes particuliers " 7 .

Le cadre institutionnel de la légitimité démocratique semble être constitué par le concours de ces deux seuls pouvoirs entre une volonté politique et une force d'application ${ }^{8}$. Rousseau lui-même précise que " c'est de l'effet

4. Contrat Social, III, 1, t. III, p. 395. Toutes les références à l'œuvre de Rousseau renverront, sauf mention particulière, aux CEuvres Complètes des éditions de la Pléiade.

5. Ibid.

6. Lettres écrites de la montagne, Lettre 6, p. 807. Nous sommes en présence, dans le Contrat Social, d'une légitimité démocratique qui se fonde sur trois idées essentielles :

$1^{\circ}$ La souveraineté du peuple signifie pour Rousseau que le peuple dispose d'une réelle participation à l'activité législative : "Le Peuple soumis aux lois en doit être l'auteur ». (Contrat Social, II, 6, p. 380.)

$2^{\circ}$ Les droits ne sont pas fixés par des décrets ou par l'arbitraire d'une volonté particulière, ils sont le contenu positif des lois : " Dans l'état civil tous les droits sont fixés par la loi ». (Ibid. p. 378.)

$3^{\circ}$ Etant créé par les lois, le droit produit à son tour l'égalité. « Le pacte social établit entre les citoyens une telle égalité qu'ils doivent jouir tous des mêmes droits. Ainsi par la nature du pacte, tout acte de souveraineté, c'est-à-dire tout acte authentique de la volonté générale, oblige ou favorise également tous les citoyens [...] ». (Ibid. II, 4, p. 374).

7. Lettres écrites de la montagne, Lettre 6, t. III, p. 808.

8. On ne peut pas affirmer que le Législateur, dans le Contrat Social, appartienne au dispositif institutionnel et qu'il soit en tant que tel un pouvoir de l'État. En effet, Rousseau souligne qu'il n'est " point magistrature et qu'il n'est point souveraineté ». (Contrat Social, II, 7, p. 382). Le Législateur possède une place politique tout à fait particulière puisqu'il n’appartient ni à la puissance législative, ni à la puissance exécutive. 
combiné de ce concours (celui du législatif et de l'exécutif) que résulte le jeu de toute la machine " politiques ${ }^{9}$. Pourtant, en dépit même de cette dernière affirmation, Rousseau en appelle à la nécessité d'un troisième pouvoir situé en tiers entre le pouvoir législatif et le pouvoir exécutif : "le Tribunat ». À cet effet, il souligne :

Quand on ne peut établir une exacte proportion entre les parties constitutives de l'État, ou que des causes indestructibles en altèrent sans cesse les rapports, alors on institue une magistrature particulière qui ne fait point corps avec les autres, qui replace chaque terme dans son vrai rapport [...]. Ce corps je l'appellerai Tribunat ${ }^{10}$.

Ce deuxième texte nous introduit bien au problème du Contrat Social. En effet, alors que dans le texte précédent, Rousseau semble affirmer que l'analyse des institutions est achevée et que la légitimité du politique est constituée par le pouvoir législatif et le pouvoir exécutif, dans ce passage du livre IV, dernier livre du Contrat Social, il affirme la nécessité d'un troisième pouvoir dans l'État. La légitimité du politique ne peut plus uniquement se déterminer par "le concours de deux pouvoirs".

Alors, pourquoi la légitimité politique, qui semblait se jouer dans les termes de la volonté et de la force, du Souverain et du Gouvernement, en appelle-t-elle, avec le Tribunat, à la nécessité d'un troisième pouvoir ? À quelle nécessité politique renvoie-t-il ?

Rousseau répond en ces termes à ces deux questions : " le Tribunat ne pouvant rien faire, peut tout empêcher ${ }^{11}$. La nécessité du Tribunat renvoie à la nécessité politique de créer une institution dont la seule fonction est d'être un moyen d'empêcher une certaine dynamique des institutions politiques.

Pour comprendre cette dynamique politique et la nécessité corrélative du Tribunat, il faut préciser le fait que, chez Rousseau, le rapport entre le législatif et l'exécutif repose sur un principe politique particulier. Le principe central n'est pas celui de la séparation des pouvoirs, si cher à Montesquieu, dont le but est d'assurer leur mutuelle limitation. Le Contrat Social ordonne le rapport entre les pouvoirs à partir d'une stricte subordination de l'exécutif au législatif ${ }^{12}$. Le sort de la légitimité politique dépend du respect et du maintien de cette subordination : si « la subordination venait à manquer entre ces deux pouvoirs, le pouvoir exécutif ne dépendrait plus du pouvoir législatif et

9. Ibid.

10. Contrat Social, IV, 5, p. 454.

11. Contrat Social, IV, 5, p. 454.

12. Nous sommes en accord avec l'interprétation de Derathé. Il refuse, en effet, de faire de «Rousseau un partisan de la séparation des pouvoirs et un disciple de Montesquieu ». (J.J. Rousseau et la science politique de son temps, Paris, Vrin, 1979, p. 300). Pour Derathé, dans le Contrat Social, " il ne s'agit aucunement de donner une indépendance quelconque à l'exécutif ", mais bien d'établir « une subordination de l'exécutif au législatif ». (Ibid. p. 301). 
l'exécution n'aurait aucun rapport nécessaire aux lois ; la loi ne serait qu'un mot, et ce mot ne signifierait plus rien $»^{13}$.

Pour Rousseau, la légitimité politique suit nécessairement le destin parallèle de la loi et de la liberté : « la liberté suit toujours le sort des Lois, elle règne ou périt avec elles ${ }^{14}$. Le sort des lois et de la liberté est déterminé par la longévité politique du principe de subordination, c'est-à-dire par la possibilité de "maintenir le gouvernement dans la dépendance des lois ${ }^{15}$.

Précisément, ce principe de subordination est constitutionnellement fragile, fragilité due à une dynamique particulière du gouvernement :

Comme la volonté particulière agit sans cesse contre la volonté générale, ainsi le gouvernement fait un effort continuel contre la souveraineté. Plus cet effort augmente, plus la constitution s'altère, et comme il n'y a point ici d'autre volonté de corps qui résistant à celle du Prince face équilibre avec elle, il doit arriver tôt ou tard que le Prince opprime enfin le souverain et rompe le traité social $^{16}$.

De cette citation, il convient de souligner deux idées :

$1^{\circ}$ - C'est bien une institution légitime et nécessaire, le Gouvernement, qui met en échec la légitimité du politique. Nous sommes en présence d'une idée tout à fait importante pour penser le politique : c'est bien le politique lui-même par le jeu de ses institutions légitimes et nécessaires qui produit sa propre illégitimité ${ }^{17}$. Cette illégitimité est produite par la dynamique du gouvernement «qui tend fortement et continuellement à subjuguer la puissance législative ${ }^{18}$. La politique, d'abord en tant que pensée, ensuite en tant qu'action, doit affronter la limite de sa propre légitimité, constituée par cette dynamique gouvernementale.

$2^{\circ}$ - Cette limite est, peut-être, une invitation à lire le Contrat Social comme un problème de temps : si le gouvernement « tend continuellement à subjuguer la puissance législative » et à renverser sa subordination au législatif, comment faire durer ce principe de subordination ? Voilà ce qui est, pour nous, l'un des problèmes de la légitimité du Contrat Social. En

13. Lettres écrites de la montagne, Lettre 7, p. 826.

14. Ibid. Lettre 8, p. 842.

15. Ibid. Lettre 8, p. 842.

16. Contrat Social, III, 10, p. 421.

17. On peut souligner l'originalité de la pensée de Rousseau qui nous fait sortir d'une certaine " innocence » de la représentation de la légitimité politique. Pour nombre de penseurs politiques, l'échec de la légitimité politique renvoie à des causes externes, étrangères aux institutions légitimes et à l'esprit des lois de la légitimité. La légitimité périt par la rencontre d'accidents qui viennent corrompre son principe : « les gouvernements périssent parce que des accidents particuliers en violent le principe ». (Montesquieu, De l'esprit des Lois, VIII, 10, G.F, t. I, p. 250). Pour Rousseau, la légitimité politique ne doit pas se préserver seulement des accidents, mais surtout d'elle-même, car ce sont ses propres institutions qui créent une dynamique politique illégitime.

18. Rousseau, Considérations sur le gouvernement de Pologne, t. III, p. 977. 
caractérisant le gouvernement comme l'opérateur d'une dynamique de renversement de la légitimité politique, Rousseau affirme que la politique repose sur le principe de subordination de l'exécutif au législatif qui est constitutionnellement provisoire et fragile. Dans le texte du livre III du Contrat Social, Rousseau donne l'une des raisons de cette fragilité du principe de subordination : l'absence d'une "volonté de corps qui résiste au gouvernement et face équilibre avec elle ». Précisément, Rousseau ne définit-il pas le Tribunat comme " une magistrature particulière qui replace chaque pouvoir dans son vrai rapport ", c'est-à-dire dans celui de la subordination ${ }^{19}$ ?

On peut donner la raison de l'écart entre nos deux textes de départ. Entre l'affirmation qu'il n'existe dans « le jeu de la machine politique » que deux pouvoirs et la nécessité d'en appeler avec le Tribunat à un troisième pouvoir, il n'y a pas contradiction. Rousseau interroge, dans le Contrat social, la légitimité politique dans deux perspectives différentes, mais complémentaires.

La première s'interroge sur les institutions politiques donnant fondement et vie à l'État. Ce fondement et cette vie se déterminent par le concours du Souverain et du Gouvernement, sans lequel il n'y aurait légitimité des lois ni dans leur production, ni dans leur application.

La deuxième éclaire le principe sur lequel doit reposer le rapport légitime entre le pouvoir législatif et le pouvoir exécutif. Ce rapport est celui de la subordination de l'exécutif au législatif.

Par conséquent, la légitimité politique est assurée, non seulement quand il existe un pouvoir législatif démocratique et un pouvoir exécutif donnant réalité aux lois, mais aussi quand la subordination des pouvoirs est réalisée. C'est cette réalisation qui nécessite la création et l'intervention d'un tiers pouvoir. C'est bien en raison d'une fragilité constitutionnelle de ce principe de subordination, que Rousseau réfléchit à des moyens politiques conférant une durée politique à ce principe essentiel de la légitimité politique ${ }^{20}$. Dans le Contrat Social, Rousseau, tout en se livrant à une analyse de la légitimité politique dans les termes de la volonté générale et de la loi, détecte les points de retournement de cette légitimité en son contraire.

19. Contrat Social, IV, 5, p. 454.

20. Il existe bien complémentarité, dans le Contrat Social, entre les deux approches de la légitimité politique. Toute légitimité politique est une théorie des pouvoirs constitutifs de L'État. Mais Rousseau introduit l'idée que la légitimité ne repose pas seulement sur une comptabilité des pouvoirs essentiels, mais sur le principe qui les dirige. Selon lui, c'est la subordination de l'exécutif qui assure la liberté politique, car elle garantit la participation des citoyens au pouvoir législatif. En effet, l'échec du principe de subordination des pouvoirs signifie qu'il « ne reste dans l'État que la seule puissance exécutive. La puissance exécutive n'est que la force, et où règne la seule force l'État est dissous ». (Lettres écrites de la montagne, Lettre 7, p. 815). Conférer de la durée politique au principe de la subordination des pouvoirs par des moyens politiques est tout aussi important, pour la légitimité du Contrat Social, que la définition du Souverain et de la volonté générale. 
Dans cette perspective, le problème du Contrat Social se pose alors en ces termes : à partir du moment où le gouvernement est, d'une part, une institution légitime et nécessaire et, d'autre part, l'opérateur de la mise en échec du principe de subordination, existe-t-il des moyens politiques susceptibles d'être des obstacles à sa dynamique de renversement de la légitimité politique ?

Rousseau propose trois moyens institutionnels qui évitent cette dynamique du gouvernement en donnant une durée politique au principe de subordination :

$1^{\circ}$ l'existence du Tribunat,

$2^{\circ}$ la possibilité, pour le citoyen, de contrôler le Gouvernement,

$3^{\circ}$ une théorie de la circulation du pouvoir ${ }^{21}$.

Ces trois moyens institutionnels inscrivent la légitimité politique dans la perspective de la résistance. L'intérêt et la valeur politique du Contrat Social ne résident pas simplement dans le fait qu'il prend soin de distinguer le Souverain du Gouvernement ${ }^{22}$, mais aussi dans la détermination de moyens politiques permettant, d'une part, de " prévenir les usurpations du gouvernement ${ }^{23}$ et, d'autre part, d' " unir la liberté au principe de subordination et de maintenir le Gouvernement dans la dépendance des lois ${ }^{24}$.

En déterminant ces moyens, Rousseau affirme qu'une légitimité politique doit se mettre en mesure de répliquer à la création de sa propre illégitimité toujours initiée par la dynamique d'institutions légitimes et nécessaires. Il définit lui-même cette légitimité par la notion de résistance : " les entreprises du gouvernement échouent quand elles trouvent de la résistance $»^{25}$. On peut définir cette politique de la résistance comme une politique négative, en la rapprochant de la définition rousseauiste de «l'éducation négative ». À son sujet, Rousseau affirme : la «bonne éducation doit être purement négative, elle doit consister, non à guérir les vices du cœur humain [...], mais à les empêcher de naître ${ }^{26}$. De même, la politique négative a pour but

21. Ces trois moyens politiques ne sont pas tous définis dans le Contrat Social, seul le premier, le Tribunat, appartient à cet ouvrage. Les deux autres sont définis respectivement dans les Lettres écrites de la montagne et dans les Considérations sur le Gouvernement de Pologne. On pourrait alors nous reprocher la création de liens factices entre ces textes. Nous ne le pensons pas. Il nous semble, au contraire, qu'ils acquièrent " un gain » de sens si on les interprète comme des réponses au problème de la subordination de l'exécutif, initié par le Contrat Social.

22. Rousseau souligne que cette distinction est très importante pour réfléchir la légitimité politique. Rousseau en fait même une modalité de lecture du Contrat Social, puisqu'il conseille "de lire avec quelque soin dans le Contrat Social les deux premiers chapitres du livre troisième ", (Lettres écrites de la montagne, Lettre 5, p. 771), livres où il définit le Gouvernement comme puissance exécutive.

23. Contrat Social, II, 18, p. 434.

24. Lettres écrites de la montagne, lettre 8, p. 850.

25. Ibid. Lettre 7, p. 816.

26. Roussean Juge de Jean-Jacques, Dialogue 1, t. I, p. 687. 
d'empêcher la naissance de l'illégitimité politique résultant de la mise en échec, par le gouvernement, du principe de subordination.

Il nous faut examiner les trois moyens institutionnels, pensés par Rousseau, pour donner une durée politique au principe de subordination.

\section{Le premier moyen institutionnel : le Tribunat.}

En caractérisant le Tribunat comme étant le premier moyen institutionnel, nous ne voulons pas signifier qu'existe chez Rousseau un ordre hiérarchique au sein de cette politique négative. Il s'agit, bien plutôt, d'un dispositif qui n'est efficace que par la réunion de ces trois moyens institutionnels.

En soulignant l'origine et la fonction romaines du Tribunat, " protéger le Souverain contre le Gouvernement ${ }^{27}$, Rousseau en mesure tout la positivité politique pour Rome:

L'Auteur, qui se plait à vous présenter de grands exemples, vous offre celui de

l'ancienne Rome. Il lui reproche avec dédain ses tribuns brouillons et séditieux : il déplore amèrement sous cette orageuse administration le triste sort de cette malheureuse ville, qui pourtant n'étant rien encore à l'érection de cette magistrature, eut sous elle cinq cents ans de gloire et de prospérités et devint la capitale du monde ${ }^{28}$.

Ce texte nous rappelle le jugement que porte Machiavel sur cette institution : "Si les troubles de Rome ont occasionné la création des tribuns, on ne saurait trop les louer [...]. Ils furent établis comme les gardiens les plus assurés de la liberté romaine ${ }^{29}$.

Pour ces deux auteurs, le Tribunat appartient à la légitimité du politique, car il assure le rôle de "protecteur du souverain » ou de "gardien le plus sûr de la liberté ». Cette communauté de jugement sur le Tribunat vient de la prise en compte d'une instabilité inhérente au politique.

Pour Machiavel, le sol où s'enracine l'autorité politique est instable car il est irrémédiablement traversé par deux désirs contradictoires. « Dans toute cité, on trouve ces deux humeurs : celle du peuple et celle des grands : le peuple ne veut pas être commandé, opprimé par les grands ; les grands veulent commander, opprimer le peuple $»^{30}$. Selon Machiavel, c'est seulement là où le conflit trouve à se manifester, c'est-à-dire là où le peuple se montre capable de résister à l'oppression des grands que la République mérite vraiment son nom. Machiavel appréhende le Tribunat comme l'institution capable de

27. Contrat Social, IV, 5, p. 454.

28. Lettres écrites de la montagne, Lettre 9, p. 879.

29. Machiavel, Discours sur la première décade de Tite-Live, I, 4, CEuvres Complètes de la Pléiade, Paris, Gallimard p. 391. Il ne semble guère concevable que ce texte de Machiavel ait été inconnu de Rousseau, Machiavel étant l'un des auteurs les plus cités du Contrat Social.

30. Le Prince, IX, Euvres Complètes de la Pléiade, Paris, Gallimard, p. 317. 
provoquer cette résistance en donnant au désir du peuple un débouché public et institutionnel ${ }^{31}$.

Pour Rousseau, l'instabilité propre à la légitimité politique ne vient pas de la contradiction de deux désirs, mais de l'asymétrie de puissance entre les institutions fondamentales du Contrat Social, le Souverain et le Gouvernement. La légitimité politique est un rapport entre des institutions qui ont des puissances asymétriques : en lui-même, le Souverain ne dispose pas de la force, puisqu'il n'est qu'une volonté législative et, en lui-même, le Gouvernement n'est qu'une puissance d'application des lois. Par conséquent, la force et la puissance appartiennent au seul Gouvernement. Il nous semble, alors important, de souligner cette idée. Rousseau instaure une différence essentielle entre le Souverain et le Gouvernement, différence qui est la condition de possibilité de la rectitude de la volonté générale : "Il n'est pas bon que celui qui fait les lois les exécute, ni que le corps du peuple détourne son attention des vues générales pour la donner aux objets particuliers ${ }^{32}$. En étant le seul détenteur de la force publique, le gouvernement empêche que les citoyens se détournent " des vues générales ». Mais, tout en légitimant l'asymétrie de puissance entre le Souverain et le Gouvernement, Rousseau en mesure tous ses effets politiques négatifs. En effet, comment éviter, " s'il arrivait enfin que le Prince eut une volonté particulière plus active que celle du Souverain, et qu'il usât pour obéir à cette volonté particulière de la force publique qui est dans ses mains ", d'avoir dans l'État "deux souverains, l'un de droit et l'autre de fait » ${ }^{33}$.

Du fait de cette asymétrie entre la volonté du Souverain et la puissance du gouvernement, la légitimité politique, laissée à elle-même, produit sa propre illégitimité, par une dynamique de concentration du pouvoir dont le Gouvernement est l'opérateur. Rousseau nous présente ainsi cette dynamique :

Le Peuple Souverain charge quelques-uns de ses membres d'exécuter ses volontés. Ces Officiers, après avoir rempli leur commission en rendent compte, et rentrent dans la commune égalité. Peu à peu ces commissions deviennent fréquentes, enfin permanentes. Insensiblement il se forme un corps qui agit toujours. Un corps qui agit toujours ne peut pas rendre compte de chaque acte [...] ; bientôt il vient à bout de n'en rendre d'aucun. Plus la puissance qui agit est active, plus elle énerve la puissance qui veut [...]. Enfin l'inaction de la puissance qui veut la soumet à la puissance qui exécute ; celle-ci rend peu à peu ses actions indépendantes, bientôt ses volontés: au lieu d'agir pour la puissance qui veut, elle agit sur elle. Il ne reste alors dans l'État qu'une puissance agissante, c'est l'exécutive. La puissance exécutive n'est que la force, et où règne la seule force l'État est dissous ${ }^{34}$.

Dans ce texte exemplaire, Rousseau définit l'asymétrie politique entre " la puissance qui veut », le Souverain et « la puissance qui exécute », le

31. Discours sur la première décade de Tite-Live, I, 7, p. 400.

32. Contrat Social, III, 1, p. 399.

33. Ibid.

34. Lettres écrites de la montagne, lettre 7, p. 815. 
Gouvernement. Nous sommes bien en présence d'un pouvoir qui n'est que volonté et d'un pouvoir qui n'est que force. Rousseau présente ici l'asymétrie de ces deux institutions légitimes du politique par un processus d'autonomisation du Gouvernement.

Rousseau affirme bien que le Gouvernement occupe peu à peu la totalité du champ politique, "en rendant indépendantes ses actions et ses volontés ». Il devient alors le seul et unique pouvoir permanent du politique : " il ne reste alors dans l'État qu'une puissance agissante, c'est l'exécutive ». Le résultat de ce processus est une métamorphose des fins du politique. La seule fin qui vaille est celle du seul pouvoir agissant : la force, « la puissance exécutive n'est que la force ».

De plus, il faut souligner que ce texte reste fidèle aux enseignements du Contrat Social, car il souligne la fragilité constitutionnelle du principe de subordination. Rien dans le processus de cette Lettre 7 des Lettres écrites de la montagne ne fait obstacle à la dynamique du Gouvernement et n'assure au principe de subordination une longévité politique. Au terme du processus, le Gouvernement, de pouvoir subordonné, est devenu un pouvoir indépendant : " au lieu d'agir pour la puissance qui veut, le gouvernement agit sur elle ». Il existe bien chez Rousseau cette idée que les deux institutions politiques légitimes, laissées à elles-mêmes, produisent nécessairement une asymétrie du pouvoir et de la puissance conduisant à la disparition de l'État.

Par conséquent, prendre ce problème au sérieux revient à donner au principe de subordination une consistance politique en empêchant l'occupation du pouvoir politique par une seule institution. Le Tribunat se présente comme un tel obstacle, et en ce sens, il n'est efficace qu'en tant qu'institution de la négativité. Il n'a d'autre raison d'être que de faire obstacle à la dynamique du Gouvernement.

Mettre en relief la nature de cet obstacle, c'est souligner cette idée importante du Contrat Social : si, chez Rousseau, il n'y a pas lieu de parler d'un équilibre ou d'un "équilibrage » des pouvoirs, il y a nécessité à parler, pour le Gouvernement, d'un pouvoir équilibré. Le pouvoir exécutif est légitime en vertu de son point d'équilibre politique entre la rectitude de sa volonté et la latitude de sa puissance. La détermination de ce point d'équilibre relève de l'art politique, " art qui est de savoir fixer le point où la force et la volonté du gouvernement, toujours en proportion réciproque, se combinent dans le rapport le plus avantageux à l'État ${ }^{35}$.

35. Contrat Social, II, 2, p. 402. Ce point d'équilibre entre la force et la volonté du Gouvernement donne contenu au principe de subordination : il manifeste que la force et la volonté gouvernementales obéissent aux directives de la volonté générale. Mais, ce point d'équilibre explique la fragilité de ce même principe de subordination. La moindre variation de la proportion entre la force et la volonté au sein du Gouvernement annule la subordination de l'exécutif et favorise la concentration du pouvoir. 
Avec ce point d'équilibre, nous sommes en présence du point névralgique de la légitimité politique, car le "Gouvernement tend toujours à renforcer sa puissance $»^{36}$ et, par conséquent, à détruire « la proportion réciproque » entre sa force et sa volonté. Précisément, la nécessité du Tribunat est appelée par un problème de "proportion ». Le Tribunat est la réponse que se donne la légitimité politique pour résoudre cet état de crise qui apparaît quand « on ne peut plus établir une exacte proportion entre les parties constitutives de l'État ${ }^{37}$. Si la cause de l'altération, dans la proportion des institutions, est due à la rupture du point d'équilibre, au sein du gouvernement, entre sa force et sa volonté, ce point d'équilibre est précisément le lieu d'intervention du Tribunat. La fonction politique du Tribunat est moins de fixer le point d'équilibre entre la force et la volonté du Gouvernement, que de faire obstacle à toute variation de la proportion entre ces deux éléments constitutifs du gouvernement.

On peut nous objecter que Rousseau définit une autre nécessité concernant l'intervention politique du Tribunat puisqu'il affirme explicitement : "Le Tribunat sert quelquefois à protéger le Souverain contre le gouvernement $[\ldots]$, quelquefois à soutenir le Gouvernement contre le peuple $[\ldots] »^{38}$. Rousseau relie le Tribunat à une double nécessité : être un obstacle soit à la puissance excessive du gouvernement, soit à la volonté souveraine du peuple. La question que nous poserons à cette double nécessité est la suivante : si le Tribunat répond à deux problèmes politiques, possèdent-ils tous deux le même coefficient de gravité politique ? L'autre question qu'il convient de se poser est : existe-t-il un texte dans lequel Rousseau compare et mesure la gravité respective de l'abus de pouvoir gouvernemental et de la crise de la souveraineté ? Un tel texte existe, où Rousseau affirme qu'il faut, en politique, départager les maux terribles qui, une fois installés, sont sans fin, des maux qui, mêmes s'ils sont importants, sont passagers. Donnons, par conséquent, la parole au philosophe genevois :

Tout abus est un mal, souvent inévitable, pour lequel on ne doit pas proscrire ce qui est bon en soi. Mais comparez, et vous trouverez d'un côté des maux sûrs, des maux terribles sans borne et sans fin ; de l'autre l'abus même difficile, qui s'il est grand sera passager, et tel, que quand il a lieu il porte toujours avec lui son remède. [...] Mais supposons cet abus de la liberté aussi naturel que l'abus de puissance. Il y a toujours cette différence entre l'un et l'autre, que l'abus de liberté tourne au préjudice du peuple qui en abuse, et le punissant de son propre tort le force à en chercher le remède ; ainsi de ce côté le mal n'est jamais qu'une crise, il ne peut être un état permanent. Au lieu que l'abus de la puissance ne tournant point au préjudice du puissant mais du faible, est par sa nature sans mesure, sans frein, sans limites ${ }^{39}$.

36. Lettres écrites de la montagne, Lettre 6, p. 808.

37. Contrat Social, IV, 5, p. 453.

38. Ibid. p. 454.

39. Lettres écrites de la montagne, lettre 9, p. 890-891. 


\section{$334 \cdot$ Philosophiques / Automne 2000}

Le Gouvernement et le Souverain produisent respectivement des maux politiques. Mais Rousseau les distingue, dans ce texte, par leurs conséquences politiques. Ainsi, si tout abus de pouvoir est un mal, cela ne signifie pas qu'entre l'abus produit par le souverain et celui produit par le Gouvernement «il y ait égalité dans leurs conséquences » ${ }^{40}$. Cette différence, Rousseau la présente, dans notre passage, par la distinction entre les notions de " crise » et «d'abus de puissance ». Seul le gouvernement relève de ce dernier phénomène politique.

La crise du souverain apparaît quand son activité législative ne correspond plus aux principes qui lui ont donné naissance, c'est-à-dire quand le peuple légifère sur un objet particulier et non plus sur l'intérêt général. Le peuple « en jugeant ce qui lui est étranger n'a plus aucun vrai principe d'équité qui le guide ${ }^{41}$. Cela entraîne le peuple à vouloir appliquer luimême les lois ${ }^{42}$. En abusant de sa liberté législative et en la faisant porter sur des objets qui lui sont étrangers, le peuple porte préjudice à lui-même. C'est ici que doit se mesurer la conséquence politique de l'abus de la liberté législative. Celui-ci ne profite à personne. Au contraire, c'est le peuple tout en entier qui en supporte les désagréments politiques. Rousseau, alors, dit que les citoyens, en cherchant " eux-mêmes le remède " à ce mal politique, évitent qu'il ne devienne "un état permanent». En revanche, le mal politique produit par le gouvernement n'est pas solidaire d'une crise passagère du politique. L'abus du pouvoir gouvernemental a pour conséquence de créer des bénéficiaires politiques de ce mal politique. Là est toute la différence entre les deux maux de l'État. Si, dans le premier cas, la condition politique devient onéreuse pour l'ensemble des citoyens, dans le second cas, le préjudice politique ne concerne que le "faible ». La conséquence politique majeure de l'abus de puissance est de créer une dissymétrie entre le puissant et le faible. Il change les termes du politique, car il en modifie les acteurs. L'espace politique n'est plus constitué par des citoyens disposant d'une égale puissance de participation à la législation, mais par des individus, qui, en disposant d'un coefficient de puissances politiques variables, se distribuent autour des catégories de puissants ou de faibles. Rousseau précise qu'à partir du moment où le mal politique crée des bénéficiaires de l'abus du pouvoir, il ne connaît plus aucune limite. Le gouvernement, par son abus de pouvoir, usurpe la souveraineté et transforme la finalité des lois en défense de privilèges.

Il ne nous semble pas utile d'énumérer les différentes formes que peut revêtir l'abus de pouvoir propre au gouvernement. Plus essentiel, nous semble$\mathrm{t}$-il, est d'insister sur sa nature et ses conséquences politiques. Sa nature ne ren-

40. Ibid. p. 890

41. Contrat Social, II, 4, p. 473.

42. Rousseau dit «Il n'est pas bon que celui qui fait les lois les exécute ». (Contrat Social, III, 4, p. 404). Si le peuple souverain exécute les lois, le risque est grand que le fait et le droit se confondent et que la loi perde sa généralité. 
voie pas simplement à la soif de pouvoir d'un individu qui confondrait l'ordre de ses désirs avec l'ordre du politique. L'abus gouvernemental du pouvoir correspond à la création d'une volonté politique indépendante des citoyens. Cette volonté, conséquence d'une cristallisation d'intérêts particuliers de différents groupes sociaux, a pour conséquence de détruire l'égalité politique et de dénaturer la loi. Rousseau présente ainsi cette volonté du gouvernement, une fois dirigée par l'intérêt particulier de ces groupes: loin d'être une simple force d'exécution de la volonté générale, le Gouvernement « est sans cesse attentif à marquer des distances trop peu sensibles dans ses égaux de naissance, il ne voit en eux que ses inférieurs, et brûle d'y voir des sujets. Armé de toute la force publique [...], interprète et dispensateur des lois qui le gênent, il s'en fait une arme offensive et défensive ${ }^{43}$. Avec cette double conséquence de l'abus gouvernemental, Rousseau nous sensibilise à une détermination essentielle de la démocratie. En effet, cette caractérisation de l'abus politique par la formation d'intérêts indépendants et particuliers est une mise en garde de la fragilité de la volonté politique démocratique. Si cette volonté se définit par sa généralité, elle court toujours le risque d'être investie par des intérêts particuliers qui, en prenant le masque de la généralité, défend des intérêts privés. Rousseau a tout à fait conscience de cette fragilité quand il nous dit: « La voix du peuple est la voix de Dieu ; mais malheureusement cette voix sacrée est toujours faible dans les affaires contre le cri de la puissance ${ }^{44}$. Précisément, la connaissance de cette faiblesse de la voix du peuple enjoint Rousseau de penser à des moyens politiques lui donnant consistance.

Ainsi, grâce au passage de la Neuvième lettre écrite de la montagne, on comprend que, non seulement Rousseau produit une différence des maux politiques par leurs conséquences, mais aussi qu'il hiérarchise la nécessité de recourir au Tribunat.

Rousseau affirme bien que le Tribunat doit protéger le Gouvernement du peuple. Mais si les maux causés par le Souverain ne sont que des crises passagères, l'action du Tribunat ne sera jamais importante et décisive. L'action décisive du Tribunat concerne les maux du Gouvernement qui sont "sans frein et sans limites ». Ce sont bien ces maux qui fragilisent la volonté générale et, par là même, la démocratie. Par conséquent, c'est bien lorsque le Tribunat "protège le Souverain contre le Gouvernement ", que son action est déterminante. Par conséquent, il est l'institution qui doit précisément empêcher que l'abus de puissance ne devienne " sans mesure, sans frein, sans limites ». Le Tribunat possède cette capacité politique, car il est un régulateur de la puissance gouvernementale.

Le Tribunat, nous dit Rousseau, "replace chaque terme dans son vrai rapport » et dans leur « exacte proportion ${ }^{45}$.

43. Lettres écrites de la montagne, Lettre 9, p. 889.

44. Ibid. Lettre 8, p. 862.

45. Contrat Social IV. 5, p.453. 
Il faut dissocier ici les termes de "rapport » et de «proportion » qui ne répondent pas à la même réalité politique de la légitimité. En effet, le terme de « rapport » définit la relation constitutionnelle qu'entretiennent le Souverain et le Gouvernement. Ce " vrai rapport » est celui du principe de la subordination de l'exécutif au législatif. Précisément, le Tribunat lui confère une consistance et une durée du fait même de la position particulière qu'il occupe dans l'espace du Contrat Social. Ce n'est pas sans raison que Rousseau affirme qu'il est « un moyen terme entre le Gouvernement et le Souverain ${ }^{46}$. De cette position particulière, Rousseau en déduit deux effets politiques importants. En étant situé en tiers entre le Souverain et le Gouvernement, le Tribunat est, d'une part le «conservateur des lois et du pouvoir législatif » et, d'autre part, «le protecteur du Souverain contre le Gouvernement ${ }^{47}$.

À l'égard de ce dernier effet, Rousseau précise que tel était la fonction du Tribunat à Rome : « il sert à protéger le souverain contre le Gouvernement, comme faisaient à Rome les Tribuns du peuple ${ }^{48}$. Le rappel par Rousseau de l'origine et de la fonction romaines du Tribunat n'est pas le signe d'une nostalgie politique. Il manifeste l'idée que, par son origine même, le Tribunat est une institution qui répond à une tension entre deux pouvoirs aux puissances asymétriques ${ }^{49}$. Le Tribunat est un pouvoir conservateur, car il empêche que le déséquilibre de puissance entre le Souverain et le Gouvernement enclenche la dynamique d'usurpation de la souveraineté. Il peut être un tel obstacle du fait qu'il est " un modérateur de la puissance exécutive ${ }^{50}$. Il dispose donc de la faculté politique de modérer les variations de puissance.

Par cette caractéristique, on comprend la raison pour laquelle le Tribunat peut établir et surtout conserver « les proportions » de puissance des institutions politiques. Ce terme n'a pas pour but de caractériser la relation entre le Souverain et le Gouvernement, il a pour fonction de déterminer l'équilibre qui doit exister au sein du Gouvernement, entre sa volonté et sa

46. Ibid. p. 454.

47. Ibid.

48. Ibid.

49. On peut faire référence une nouvelle fois à Machiavel pour cette caractérisation du Tribunat. À cet effet, il affirme "qu'après bien des tumultes et des périls occasionnés par les excès auxquels se portèrent les deux ordres (le sénat et le peuple), on en vint pour la sûreté $d u$ dernier (le peuple) à la création des tribuns, et on leur accorda tant de prérogatives, [...], qu'ils formèrent entre le Sénat et le peuple une barrière qui s'opposa à l'insolence des premiers ». (Discours sur la première décade de Tite-Live, I, 3, p. 389). Cette définition machiavélienne du Tribunat est partagée par Rousseau. Il est le moyen politique qui donne une réponse institutionnelle aux déséquilibres entre le peuple souverain et le pouvoir exécutif. L'analyse des institutions romaines donne à Rousseau le modèle d'une institution qui est conçue, dès son origine, comme une régulation des tensions de puissance entre deux pouvoirs politiques. On peut souligner que le livre IV du Contrat Social est une réflexion politique d'inspiration romaine car Rousseau analyse quatre institutions importantes de Rome : Les Comices romains, chapitre IV, le Tribunat, Chapitre V, la Dictature, chapitre VI et la censure, chapitre VII.

50. Contrat Social, IV, 5, p. 455. 
force politiques. L'institution gouvernementale pose, au politique, le problème d'une contradiction entre sa structure et sa dynamique politiques. Par sa structure, il est une proportion entre sa volonté politique et sa force exécutive. Par sa dynamique, le Gouvernement tend à rompre l'équilibre entre sa volonté et sa force : le gouvernement a tendance à intensifier sa puissance.

Le Tribunat, en étant « le modérateur de la puissance exécutive ", empêche l'inflation de cette puissance. Il est, alors, un élément essentiel de "l'art politique », car il tente de maintenir « le point où la force et la volonté du Gouvernement [...] se combinent dans le rapport le plus avantageux à l'État $»^{51}$.

Le Tribunat appartient bien à ce que nous appelons la politique négative, cette politique qui naît à partir du problème que pose la dynamique du Gouvernement. Le Tribunat est constitué dans le seul but d'être un obstacle politique à cette dynamique : "le Tribunat ne peut rien faire, mais tout empêcher ${ }^{52}$. Il ne peut ni faire la loi, ni l'exécuter puisqu'il ne "doit avoir aucune portion de la puissance législative ni de l'exécutive ${ }^{53}$. Mais il peut maintenir le Gouvernement dans son « exacte proportion » entre sa volonté politique et sa force. En étant une institution " modératrice ", et, par conséquent, une institution régulatrice de la puissance du Gouvernement, le Tribunat évite que le principe de subordination ne se dissolve.

Au terme de cette étude sur le Tribunat, on pourrait légitimement critiquer notre insistance sur l'aspect exclusivement négatif du rôle du Tribunat. Sans vouloir développer une interminable justification, nous soulignerons deux traits de la pensée de Rousseau.

Premièrement, la négativité du Tribunat consiste à empêcher que le Gouvernement ne devienne le lieu d'une volonté politique indépendante et particulière. Ainsi, par sa négativité même, il est une réponse politique au problème d'une démocratie qui, tout en définissant la loi comme expression de la volonté générale, connaît la fragilité de cette généralité.

Deuxièmement, cette détermination négative doit être nuancée. Elle trouve, en effet, son complément positif avec ce que Rousseau nomme "le Droit de Représentation ». Ce droit est la possibilité pour le peuple d'intervenir politiquement, quand il fait l'expérience que la loi ne correspond plus à sa volonté d'égalité. Nous verrons que le Tribunat constitue la réponse institutionnelle de ce droit de représentation.

Il nous faut, pour cela, analyser le deuxième moyen que se donne la légitimité politique pour conférer de la durée et de la consistance au principe de subordination.

51. Ibid. II, 2, p. 402.

52. Contrat Social, IV, p. 454.

53. Ibid. 


\section{Le deuxième moyen institutionnel. Citoyenneté et inspection sur le pouvoir exécutif.}

Ce terme d'inspection appartient bien au vocabulaire de Rousseau : " Le pouvoir législatif consiste en deux choses inséparables : faire les lois et les maintenir ; c'est-à-dire, avoir inspection sur le pouvoir exécutif » ${ }^{54}$. Rousseau donne deux déterminations du pouvoir législatif qui, tout en étant " inséparables ", ne remplissent pas la même fonction politique. La première identifie le Souverain à la puissance législative, qui n'a de réalité qu'avec le peuple assemblé : "Le Souverain ne saurait agir que quand le peuple est assemblé " ${ }^{55}$. Ici, le Souverain n'est pas défini dans son rapport avec le Gouvernement, puisque précisément, il est la seule et unique puissance législative de l'État.

Ce n'est pas le cas de la deuxième détermination du Souverain, car la modalité de son action s'organise dans le rapport avec le Gouvernement qui se définit dans la perspective du problème du Contrat Social : développer une politique négative dont l'unique but est de donner une durée politique «à la subordination entre les deux pouvoirs " ${ }^{56}$. Rousseau affirme bien que "sans l'inspection du Souverain sur le pouvoir exécutif, la subordination manquerait entre ces deux pouvoirs, le dernier ne dépendrait point de l'autre, l'exécution n'aurait aucun rapport aux lois ${ }^{57}$. Il nous faut analyser la façon dont Rousseau donne affectivité à ce pouvoir politique d'inspection. Tout d'abord, il propose une définition du citoyen qui ne saurait s'arrêter à la détermination du citoyen " qui raisonne dans le silence de ses passions ${ }^{58}$. En effet, si on bloque l'interrogation rousseauiste de la politique au problème de la volonté générale, on risque fort de déterminer la cité du Contrat Social comme une cité de solitaires où les citoyens ne " doivent avoir aucune communication entre eux ${ }^{59}$, car seule cette absence de communication permet de dégager la volonté générale dans sa pureté.

Rousseau complète ainsi cette définition du citoyen présente dans le Contrat Social :

Dans un État tel que le vôtre, où la souveraineté est entre les mains du peuple, le législateur existe toujours, quoiqu'il ne se montre pas toujours. Il n'est rassemblé et ne parle authentiquement que dans le Conseil général ; mais hors du Conseil général il n’est pas anéanti ; ses membres sont épars, mais ils ne sont pas morts ; ils ne peuvent parler par des lois, mais ils peuvent toujours veiller sur l'administration des lois ; c'est un droit, c'est même un devoir attaché à leurs personnes, et qui ne peut leur être ôté dans aucun temps ${ }^{60}$.

54. Lettres écrites de la montagne, lettre 7, p. 826.

55. Contrat Social, III, 12, p. 425.

56. Lettres écrites de la montagne, lettre 7, p. 826.

57. Ibid.

58. Contrat Social, $1^{\text {ère }}$ version, I, 2, p. 286.

59. Contrat Social, II, 3, p. 371.

60. Lettres écrites de la montagne, lettre 8, p. 845. 
Dans ce passage, Rousseau distingue deux actes du citoyen. Le premier dépend de sa participation au pouvoir législatif : en étant membre du Souverain, le citoyen participe à la production du droit. Indépendamment de cette participation, le citoyen possède une autre possibilité d'intervention politique : veiller au respect institutionnel des lois. C'est bien en termes institutionnels qu'il faut interroger ce respect, car c'est bien une institution, le gouvernement, qui est animé d'une volonté de "transgresser » l'esprit des lois $^{61}$. En rompant le principe de subordination, le Gouvernement instrumentalise la loi : elle ne fixe plus des droits valant pour tous les citoyens, elle devient « une arme offensive et défensive » contre les citoyens ${ }^{62}$.

Donner une consistance au principe de subordination, c'est éviter qu'une telle instrumentalisation des lois devienne possible. Tel est le cas si dans l'exécution des lois, le Gouvernement reste "soumis au jugement du souverain parce qu'il est surveillé par le peuple ${ }^{63}$.

Cette surveillance est une détermination essentielle du citoyen, car elle est instituée par un droit que Rousseau, fidèle à la constitution de Genève, nomme «droit de représentation » dont il en fixe ainsi l'étendue :

Le législateur existant toujours voit l'effet ou l'abus de ses lois il voit si elles sont suivies ou transgressées, interprétées de bonne ou de mauvaise foi ; il y veille; il y doit veiller; cela est de son droit, de son devoir, même de son serment. C'est ce devoir qu'il remplit dans les Représentations ${ }^{64}$.

Ce droit a bien pour but d'éviter toute transgression de la loi due à une mauvaise interprétation de celle-ci par le Gouvernement ${ }^{65}$. En empêchant que le Gouvernement devienne « l'arbitre des lois » 66 , le droit de représentation est un obstacle à la création d'une politique illégitime, il appartient par conséquent à cette politique négative dont le seul but est de donner une consistance au principe de subordination. Rousseau affirme explicitement ce lien entre l'appartenance du droit de représentation à une politique négative et son effet sur le principe de subordination.

En effet, il souligne: «Le droit de représentation pris dans son vrai sens n'est lui-même qu'un droit négatif. Il consiste uniquement à empêcher la puissance exécutive de rien exécuter contre les lois ${ }^{67}$. Ce droit a bien pour

61. Ibid. lettre 9, p. 889.

62. Ibid.

63. Ibid. lettre 8, p. 843-844.

64. Ibid. p. 847.

65. Rousseau confère bien au Gouvernement le droit d'interpréter les lois, interprétation souvent nécessaire quand il faut réduire la généralité de la loi en actes particuliers : "quand la loi est équivoque c'est à l'équité du Magistrat d'en fixer le sens dans la pratique ». (Lettres écrites de la montagne, lettre 8, p. 861). Dans cette nécessité, existe le risque que le Gouvernement interprète la loi " de mauvaise foi », c'est-à-dire en la transformant " en arme offensive et défensive » contre le peuple.

66. Lettres écrites de la montagne, lettre 9, p. 892.

67. Ibid. p. 873. 
but de renforcer le principe de subordination : « Le droit de représentation [...] est le seul moyen possible d'unir la liberté à la subordination, et de maintenir le Magistrat dans la dépendance des lois ${ }^{68}$.

À l'issue de cette caractérisation du droit de représentation, Rousseau pose une question d'importance : comment éviter que ce droit soit illusoire et vain, comment éviter qu'il n'apporte à la transgression des lois " d'autre opposition, d'autre droit, d'autre résistance qu'un murmure inutile et d'impuissantes clameurs " ${ }^{69}$ ? Si, effectivement, ce droit de représentation " se borne à la rare prérogative de demander et ne rien obtenir " ${ }^{70}$, il ne donne aucune réelle consistance au principe de subordination.

Pour Rousseau, ce droit n'est pas vain car il possède « un effet réel », celui d'établir un "solide équilibre entre toutes les parties de l'État " ${ }^{71}$. Dans cette affirmation, nous retrouvons une caractérisation, rencontrée avec le Tribunat, qui est "d'établir une exacte proportion entre les parties constitutives de l'État ». Le droit de représentation renvoie lui aussi à l'idée d'une organisation du politique qui doit maintenir une proportion entre des pouvoirs aux puissances asymétriques. Le droit de représentation participe de cette mise en proportion du pouvoir du Gouvernement, proportion entre sa volonté et sa force ${ }^{72}$.

Le droit de représentation est « l'appui de la République ${ }^{73}$, car il est un principe actif de sa vie politique en donnant la possibilité aux citoyens non seulement d'intervenir dans les institutions politiques, mais de rendre l'intervention de ces institutions nécessaire pour répondre à la transgression de la loi. Le droit de représentation donne vie à une République, car, d'une part, il donne sens à l'opinion politique et, d'autre part, il a le pouvoir de provoquer l'intervention politique du Souverain et du Tribunat.

"Le Contrat Social élève l'opinion non publique au rang d'unique législateur et exclut la Publicité des discussions où le public fait usage de sa raison. [...]. Rousseau oppose l'harmonie qui doit régner au sein des assemblées aux dangereuses péroraisons des beaux parleurs » ${ }^{74}$. Cette affirmation

68. Ibid. lettre 8, p. 850. Nous rappelons que le terme de Magistrat chez Rousseau désigne les membres du Gouvernement. (Contrat Social, III, 1, p. 396).

69. Ibid. lettre 9, p. 871.

70. Ibid. lettre 8, p. 844.

71. Ibid. p. 867.

72. Si Rousseau, au sujet du droit de représentation, parle d'un « équilibre entre toutes les parties de l'État ", il ne s'agit toujours pas du principe de Montesquieu. Dans l'esprit de Rousseau, on peut parler d'un équilibre des pouvoirs, non pas en vertu de la séparation des pouvoirs, mais du fait que le Gouvernement est un pouvoir équilibré. Cet équilibre est solidaire d'une politique qui maintient «le point où la force et la volonté du gouvernement [...] se combinent dans le rapport le plus avantageux à l'État». (Contrat Social, III, 2, p. 402). En surveillant qu'en appliquant les lois, le gouvernement ne les transgresse pas, le droit de représentation est un élément essentiel de cette politique.

73. Lettres écrites de la montagne, lettre 8, p. 843.

74. J. Habermas, L'Espace Public, Archéologie de la publicité comme dimension constitutive de la société bourgeoise, Chap. IV, Paris, Payot, 1978, p. 107. 
d'Habermas, reflétant le jugement de nombreux interprètes de Rousseau, donne une idée partielle et partiale de la conception rousseauiste de l'opinion publique. Il est certain que Rousseau entretient une méfiance à l'égard de l'opinion publique pour produire la volonté générale. Mais cela ne signifie pas que sa pensée politique n'accorde aucune place à l'opinion publique et que les citoyens soient privés d'une parole publique.

Précisément, le droit de représentation permet de donner la publicité nécessaire aux observations politiques de tout citoyen. Ces observations ont pour but de contrôler le pouvoir exécutif. L'opinion des citoyens est déterminante pour rendre apparent tout abus de pouvoir produit par le Gouvernement. Avec ce droit de représentation, Rousseau affirme bien la nécessité politique d'une expression et d'une opinion publiques auxquelles chaque citoyen peut et doit participer : "l'effet des Représentations des particuliers $[\ldots]$ est de devenir enfin la voix du public $»^{75}$. Cette parole n'est pas législative, elle est " un avis » des citoyens qui doit avoir toute la publicité nécessaire. Ce droit de représentation ne dépend d'aucune arithmétique législative, il rend possible la création d'une opinion publique et d'un espace public. C'est pour cette raison que Rousseau affirme qu'avec le droit de représentation, " on ne compte pas les voix et on ne donne pas son suffrage, c'est seulement dire son avis ${ }^{76}$. Rousseau précise que cet " avis ", portant sur « la transgression des lois, devient une affaire publique $»^{77}$. Les citoyens, au moyen de " la voix du public qui s'élève ${ }^{78}$, créent un espace public qui est précisément celui de leur intervention politique. On peut compléter la définition de la République, présente dans le Contrat Social. Pour celui-ci, elle est bien « un État régi par des lois [...] où l'intérêt public gouverne, et où la chose publique est quelque chose ${ }^{79}$. Pour une République, la chose publique est quelque chose si les citoyens peuvent rendre publique leurs revendications politiques. Mais cette publicité des revendications ne constitue pas à elle seule un obstacle à la transgression des lois par le gouvernement.

Avec le droit de représentation, dont chaque citoyen est porteur, 《toute transgression des lois devient une affaire publique ${ }^{80}$. Du fait même de cette publicité, créée par les citoyens, le politique est contraint d'y apporter une réponse institutionnelle. C'est pour cette raison que Rousseau précise : le droit de représentation est "un droit de requête " ${ }^{81}$. Il poursuit ainsi cette définition " requérir n'est pas seulement demander, mais demander en vertu d'un droit qu'on a d'obtenir ${ }^{82}$. Ce droit de requête n'existe

75. Lettres écrites de la montagne, lettre 8, p. 850.

76. Ibid. p. 845.

77. Ibid p. 850 .

78. Ibid.

79. Contrat Social, II, 6, pp. 379-380.

80. Ibid.

81. Ibid, p. 846.

82. Ibid. note. 
véritablement, aux yeux de Rousseau que dans une démocratie : "C'est dans une démocratie qu'on donne authentiquement aux citoyens, aux membres du souverain, la permission [...] d'user de ce droit ${ }^{83}$. C'est seulement dans une démocratie que ce droit, d'une part, rend publics les abus de pouvoir et, d'autre part, rend nécessaire une réponse institutionnelle à la transgression des lois. Cette réponse institutionnelle développe deux conséquences importantes.

$1^{\circ}$ - L'opinion des citoyens doit se savoir écoutée du Souverain, elle doit avoir un écho qui appelle une décision politique :

L'avis des citoyens n'est que celui d'un particulier ou de plusieurs; mais ces particuliers étant membres du Souverain et pouvant le représenter quelquefois par leur multitude, la raison veut qu'alors on ait égard à leur avis, non comme à une décision, mais comme à une proposition qui la demande, et qui la rend quelquefois nécessaire ${ }^{84}$.

En rendant publique une transgression de la loi, les citoyens obligent le pouvoir d'y remédier.

$2^{\circ}$ - Cette réponse institutionnelle met en place un rapport spécifique entre le Souverain et le Tribunat. À l'égard du Souverain, le droit de représentation crée une division du travail au sein des assemblées du peuple. Il existe deux motifs politiques qui nécessitent, pour Rousseau, l'assemblée du peuple : soit légiférer, soit examiner les requêtes contenues dans les Représentations du peuple. Rousseau affirme la nécessité d'assemblées du peuple "périodiques qui se bornent aux plaintes mises en Représentations » et dans lesquelles «il n'est pas permis d'y porter aucune autre question ${ }^{85}$. À cette occasion, le Souverain examine la valeur politique de la requête déposée par les citoyens et si la transgression est avérée, il statue sur la nécessité de répondre politiquement à cet abus de pouvoir. L'application de cette décision souveraine, relevant de la particularité, ne peut être réalisée par le Souverain luimême ${ }^{86}$. Elle ne peut pas non plus être réalisée par le Gouvernement, car il est lui-même la cause de la transgression de la loi : en étant juge et partie, il n'aurait que trop tendance à "faire perdre sa force au droit de représentation ${ }^{87}$. Il faut donc recourir au troisième pouvoir, situé entre le Souverain et le Gouvernement : le Tribunat. Ce dernier, à la demande du

83. Ibid. p. 844.

84. Ibid. p. 846.

85. Ibid. p.854.

86. Le souverain ne peut remédier lui-même à l'abus de pouvoir exercé par le Gouvernement en modifiant, par exemple sa structure, car il s'agit d'un acte particulier qui est étranger à sa compétence politique : "Toute fonction qui se rapporte à un objet individuel n'appartient point à la puissance législative » (Contrat Social, II, 6, p. 379). En examinant les Représentations des citoyens, il prend la décision politique de faire obstacle à la transgression de la loi. Cette décision est déterminante car elle légitime l'intervention politique du Tribunat. 87. Ibid. p. 861. 
Souverain, régule la puissance du Gouvernement en faisant varier le nombre de ses magistrats.

Ce droit de représentation nous introduit à une tout autre logique des institutions politiques que celle relative à la législation.

La logique de la volonté générale est celle d'une "délibération des citoyens dans le silence des passions ". Cette délibération, dont le point d'aboutissement est la création de la loi, débouche sur le problème de l'application de la législation avec la nécessité du Gouvernement.

La logique instaurée par le droit de représentation a son point de départ dans la publicité donnée par les citoyens à une éventuelle transgression de la loi par le Gouvernement. Cette publicité rend nécessaire une réponse politique sous la forme d'un examen de "la plainte des citoyens par le Souverain ${ }^{88}$. Si la plainte est justifiée, le Souverain décide l'intervention politique du Tribunat auprès du Gouvernement. Ainsi, la politique négative, dont le but est de faire obstacle à la dynamique du Gouvernement, met en place un rapport institutionnel spécifique animé par un droit appartenant à chaque citoyen.

Ce droit de représentation, que Rousseau dit appartenir en propre aux démocraties, donne à cette dernière la détermination de démocratie en continu. Si cette dénomination est évidemment absente de l'œuvre de Rousseau, l'idée y est bien présente. Elle peut se résumer par cette exigence politique : les citoyens ne doivent jamais perdre le droit d'être en activité, c'est-à-dire d'avoir le droit d'intervenir dans les institutions politiques. Cette intervention ne se réduit pas, chez Rousseau, au pouvoir législatif. Elle prend aussi le visage d'un contrôle sur l'action des gouvernants, contrôle représenté par la possibilité, offerte à chaque citoyen, de rendre publique toute transgression de la loi. Les citoyens mesurent leur pouvoir, non seulement en participant à l'établissement des lois, mais en manifestant leur opinion qui rend public et apparent tout abus de pouvoir. Mais si, pour cette démocratie, l'opinion de chaque citoyen est un pouvoir, il faut qu'existe un pouvoir de l'opinion. L'opinion publique d'une telle démocratie ne signifie pas simplement le fait de s'exprimer librement sur la place publique, mais, à l'issue d'une discussion libre et publique, de rendre manifeste un abus de pouvoir auquel doit répondre une institution politique. Faire exister un pouvoir de l'opinion, c'est donner au peuple un débouché institutionnel à son opinion, qui a la possibilité de provoquer une intervention politique. Aux yeux de Rousseau, il n'y a pas de démocratie plus légitime que celle qui permet de ménager aux requêtes politiques des citoyens une issue publique, c'est-à-dire tout à la fois de leur laisser libre cours et de leur donner une réponse institutionnelle. La valeur d'une démocratie se mesure à la fois au nombre de droits politiques que possède le citoyen et aux moyens réels qu'il peut mettre en oeuvre pour mesurer que ces droits sont autant de pouvoirs

88. Ibid. p. 850. 
politiques. Ces droits possèdent un effet réel s'ils définissent, pour le citoyen, des possibilités d'intervention dans le politique.

Cette démocratie en continu multiplie les moyens pour rendre le citoyen actif en politique. Elle ne réduit pas le citoyen à son pouvoir électoral, car, aux yeux de Rousseau, on risque de réduire la portée de la liberté politique « en ne voyant la liberté que dans le droit d'élire ses chefs ${ }^{89}$.

La citoyenneté se mesure à sa possibilité de produire du politique. Dans le Contrat Social, Rousseau affirme à l'égard du politique : « ce qui n'est rien ne produit rien ${ }^{90}$. Un citoyen n'est un sujet politique que s'il produit, avec d'autres citoyens, un corps politique. Cette production ne repose pas seulement sur la possibilité de participer à la législation. Elle correspond aux possibilités effectives offertes aux citoyens à la fois de rendre publics les abus de pouvoir et d'y apporter une réelle réponse qui ne soit pas « la simple liberté de se plaindre et de ne rien obtenir $»^{91}$. Rousseau, avec le droit de représentation, réfléchit à de tels moyens politiques qui permettent aux citoyens de produire une intervention institutionnelle qui met fin à des pratiques politiques illégitimes et qui brise la dynamique de l'appropriation du pouvoir.

Ce droit de représentation tient ouverte la question de la légitimité, car il est le moyen politique dont dispose la démocratie pour répondre à la création de sa propre illégitimité ${ }^{92}$.

Il nous faut maintenant analyser le troisième moyen propre à la politique négative.

\section{Le troisième moyen institutionnel : la circulation du pouvoir.}

On peut affirmer qu'il existe, dans l'œuvre politique de Rousseau, une réelle théorie de la circulation du pouvoir. Elle est analysée dans les Considérations sur le Gouvernement de Pologne : " je voudrais que toutes les fonctions publiques menassent de l'une à l'autre; afin que nul ne s'arrange pour rester dans la sienne; ne s'en fit un métier lucratif et ne se mit au-dessus du jugement des hommes $»^{93}$.

89. Ibid. p. 838.

90. Contrat Social, II, 7, p. 363.

91. Lettres écrites de la montagne, lettre 8, p. 844.

92. Ce moyen politique de réaction et de réponse à la production de l'illégitimité politique est vraiment démocratique car tous les citoyens le possèdent et peuvent par conséquent le manifester.

93. Considérations sur le Gouvernement de Pologne, X, t. III, p. 1002. Rousseau, dans cet ouvrage, analyse l'accès au Gouvernement comme un passage graduel dans différentes fonctions publiques. Chaque passage est sanctionné par un examen de l'honnêteté politique avec laquelle le citoyen a rempli sa charge. Rousseau propose cette gradation suivante : le citoyen doit d'abord être avocat, " premier pas pour arriver aux magistratures » (Ibid. $p$. 1001), puis «servant d'État» qui lui permet d'assumer les fonctions « de commissaire à la chambre des comptes » (Ibid. p. 1021), ensuite il peut accéder au titre de «citoyen de choix » 
Dans le cadre de cet article, nous ne voulons pas analyser les différents stades qui «mènent d'une fonction publique à l'autre ». Nous nous contenterons de souligner les deux idées qui soutiennent la conception rousseauiste de la circulation du pouvoir.

La première idée appartient à la démocratie qui se caractérise, comme nous l'avons vu avec le droit de représentation, par la continuité. La valeur et la réalité d'une démocratie en continu se mesurent aux moyens politiques qu'elle met en oeuvre pour faire participer le plus grand nombre possible de citoyens au pouvoir. La circulation du pouvoir est ce moyen car, comme l'affirme Rousseau, il permet « qu'un plus grand nombre de citoyens ait part au gouvernement $»^{94}$.

Dans cette démocratie en continu, la notion de participation politique correspond point pour point à celle de citoyen. Ce dernier n'est pas seulement celui qui participe au pouvoir législatif pour s'en remettre ensuite à des professionnels de l'application des lois. Indépendamment du pouvoir législatif (puisque tous y participent), tous les citoyens, en vertu de la circulation du pouvoir, ont une égale possibilité de participer au pouvoir exécutif. Le citoyen se définit comme celui qui a la possibilité de participer au pouvoir politique, quelle qu'en soit sa nature, législative ou exécutive. À cet effet, Rousseau dit : « Nul ne doit être magistrat par état ni soldat par état. Tous doivent être prêts à remplir indistinctement les fonctions que la patrie leur impose ${ }^{95}$.

Rousseau a l'intuition que dans une démocratie, la revendication par certains citoyens «d'être des magistrats par état » ouvre la voie aux professionnels de la politique qui devient ainsi « un métier lucratif » ${ }^{96}$. Cette transformation constitue une source de pouvoir et ouvre la possibilité de sa concentration. Cette affirmation nous conduit à la deuxième idée contenue dans l'exigence rousseauiste de faire circuler le pouvoir.

Cette circulation du pouvoir appartient à la politique négative. Elle est " un moyen de prévenir les usurpations du Gouvernement " ${ }^{97}$, car elle empêche toute concentration de son pouvoir en interdisant toute permanence du pouvoir exécutif. L'affirmation rousseauiste que les membres du Gouvernement doivent avoir " une existence passagère ${ }^{98}$ est fondée sur une conviction politique.

Pour Rousseau, on ne crée pas un pouvoir politique sans créer un intérêt qui a tendance à s'écarter de la volonté générale et de s'en rendre indépendant. Ce phénomène est particulièrement sensible avec le Gouvernement, car il a tendance à rendre "sa volonté particulière plus active que celle du

qui l'autorise à exercer les fonctions " de principal de collège et d'inspecteur de l'éducation des enfants » (Ibidem) et, enfin, on peut l'élever au titre de "Custos legem », "gardien des lois » (Ibid. p. 1023) qui lui permet d'être membre du Gouvernement.

94. Fragments Politiques, Du Pacte Social, 22, p. 488.

95. Projet de constitution pour la Corse, Fragments, t. III, p. 946.

96. Considérations sur le Gouvernement de Pologne, X, p. 1002.

97. Contrat Social, III, 13, p. 434.

98. Considérations sur le Gouvernement de Pologne, VII, p. 977. 


\section{$346 \cdot$ Philosophiques / Automne 2000}

Souverain ${ }^{99}$. Fort de cette conviction, Rousseau pense que la permanence du pouvoir ne ferait qu'aggraver ce phénomène : en rendant permanente la fonction des magistrats, ceux-ci «se regardent comme les propriétaires de l'État ${ }^{100}$.

Précisément, la circulation du pouvoir est, d'une part, une prise en compte de ce phénomène et, d'autre part, une réponse politique à sa dynamique négative.

Cette circulation appartient bien à la politique négative : en rendant possible une participation étendue des citoyens au pouvoir exécutif, on empêche ses points de fixation et de concentration. Cette possibilité est le « grand ressort », le « vrai secret de l'État », car elle « empêche l'esprit d'état de s'enraciner dans un corps politique ${ }^{101}$. C'est cet enracinement qui dynamise la création d'un intérêt propre à une institution et directement contraire à l'intérêt général. Le pouvoir, qui se donne une permanence au travers de ses membres, crée un intérêt contraire à l'intérêt général préjudiciable au principe de subordination.

Précisément, nous dit Rousseau : "la puissance exécutive ainsi passagère sera plus subordonnée à la législative » ${ }^{102}$. La circulation du pouvoir a pour but de donner de la consistance au principe de la subordination des pouvoirs. Ce n'est que si "les mains du pouvoir exécutif changent » que la dynamique de la concentration du pouvoir et des intérêts rencontre un réel obstacle et que la subordination des pouvoirs connaît une solidité.

Les citoyens, membres du Gouvernement, en vertu de la circulation des charges politiques, n'assument qu'une fonction publique soumise à échéance. Rousseau insiste sur cette qualification de fonction publique. Pour lui, « le seul état permanent est celui de citoyen ${ }^{103}$, toutes les autres charges politiques que les citoyens peuvent exercer sont des fonctions publiques temporaires, "considérées comme des places d'épreuves, des degrés pour monter plus haut après l'avoir mérité » ${ }^{104}$.

La détermination rousseauiste de la fonction publique possède un double enjeu.

Le premier enjeu identifie le pouvoir à un exercice temporaire et par conséquent renouvelé. Le renouvellement fréquent des détenteurs du pouvoir exécutif rend possible une participation importante des citoyens à la politique. Du même coup, elle ouvre le politique sur une logique de la circulation et du renouvellement du pouvoir dans toutes les institutions majeures. Il doit être renouvelé, non seulement dans le Gouvernement, mais dans

99. Contrat Social, III, 1, p. 399.

100.Discours sur l'origine de l'inégalité, p. 187.

101.Considérations sur le Gouvernement de Pologne, X, p. 1000.

102.Ibid. VII, p. 977.

103.Projet de constitution pour la Corse, Fragments, p. 946.

104.Ibid. IV, p. 967. 
toutes les institutions où s'exerce une puissance politique. Rousseau le précise au sujet du Tribunat : " le meilleur moyen de prévenir les usurpations » $\mathrm{du}$ Tribunat « serait de ne pas rendre ce corps permanent $»^{105}$.

Le deuxième enjeu fonctionne sur une opposition. La fonction publique est conçue comme le négatif du professionnalisme en politique. Rousseau a l'intuition que la revendication par certains citoyens d'un titre d'expert ou de professionnel de la politique conduit à une concentration du pouvoir. Cette revendication installe la dissociation entre un peuple revêtu d'une souveraineté et l'exercice réel du pouvoir. Le terme de fonction publique veut éviter cette étrange condition politique : faire du peuple la source légitime du pouvoir, tout en l'excluant de toute participation au pouvoir.

C'est pour cette raison que Rousseau définit la fonction publique politique dans son opposition avec " un métier lucratif ${ }^{106}$. Affirmer que l'exercice du pouvoir politique relève d'une fonction publique, c'est dissocier la politique de toute idée de propriété. Il faut dissocier le pouvoir de celui qui l'exerce. Le pouvoir politique, étant celui d'une institution qui se fonde sur le principe de subordination, le membre de cette institution n'exerce, non pas le pouvoir, mais les fonctions politiques définies par cette institution. Cette idée connaît son point d'aboutissement avec l'exigence de la rotation des charges politiques. Rousseau assure ce lien entre la nature du pouvoir comme fonction publique et la nécessité de sa transmission pour "qu'un grand nombre de citoyens ait part au gouvernement ${ }^{107}$. Avec cette circulation du pouvoir rendant possible la participation des citoyens, la nature du pouvoir exécutif est une fonction publique qui ne se confond pas avec les personnes qui l'accomplissent provisoirement.

Pour conclure sur ce troisième moyen de la politique négative, on peut retenir trois caractéristiques.

1) La circulation du pouvoir, en exigeant un renouvellement périodique des magistrats exerçant le pouvoir exécutif, rend possible une participation étendue et réelle des citoyens au gouvernement.

2) Ce renouvellement fréquent, qui oblige « le passage fréquent de cette puissance exécutive par différentes mains ${ }^{108}$, donne consistance au principe de subordination car il empêche la concentration d'un pouvoir « qui rendrait ses actions et sa volonté indépendantes de la volonté générale ${ }^{109}$.

3) Ce passage du pouvoir « dans différentes mains » donne une durée au principe de subordination pour une deuxième raison. Ce changement de mains empêche toute personnalisation du pouvoir. Il refuse, avec la notion

105. Contrat Social, IV, 5, p. 455.

106.Considérations sur le Gouvernement de Pologne, X, p. 1002.

107.Fragments Politiques, Du pacte social, 22, t. III, p. 488.

108. Considérations sur le Gouvernement de Pologne, VII, p. 975.

109.Lettres écrites de la montagne, lettre 7, p. 815. 
de fonction publique, toute identification entre pouvoir personnel et pouvoir politique. Il ne peut pas se concentrer et ce pour deux raisons :

- En se transmettant, il rend possible une rotation des charges politiques entre un grand nombre de citoyens ;

- En étant une fonction publique il faut toujours dissocier celui qui exerce cette fonction et la puissance politique qui est institutionnalisée.

La circulation du pouvoir, chez Rousseau, fait partie intégrante de sa conception de la démocratie : le fait que la politique soit dévolue à tous les citoyens signifie qu'elle n'est à personne. Ce n'est qu'à la faveur de cette mobilité des citoyens dans les charges politiques, que le pouvoir est soustrait à l'appropriation. Pour Rousseau, la démocratie, loin de se réduire à l'exercice en acte de la souveraineté du peuple, exige inséparablement que personne ne puisse s'approprier un lieu de pouvoir ni par l'identification du politique à l'exercice d'un métier, ni en donnant, à celui qui l'exerce la possibilité d'une permanence. Rousseau évite ces deux écueils de la démocratie en instaurant la circulation du pouvoir dans toutes les institutions politiques.

Rousseau, à partir du problème du Gouvernement défini dans le Contrat Social, met en place trois moyens institutionnels pour faire obstacle à sa dynamique d'usurpation de la souveraineté. Pour finir de les caractériser, nous pouvons dire que, si tous trois donnent consistance au principe de subordination, ils ne se situent pas dans le même temps politique de l'usurpation gouvernementale.

Le droit de représentation et le Tribunat sont un obstacle politique à la dynamique illégitime du gouvernement, car ils répondent aux effets de la transgression de la loi. Cette réaction s'opère en deux moments : premièrement, en rendant publique cette transgression et, deuxièmement, en faisant intervenir le Tribunat, pouvoir régulateur et modérateur du gouvernement.

La théorie de la circulation du pouvoir nous renvoie aux causes de l'usurpation du gouvernement. En rendant mobiles et provisoires les détenteurs de la puissance exécutive, on empêche les points de fixation et de concentration du pouvoir qui fragilisent le principe de subordination pour, à terme, le mettre en échec.

Nous terminerons cet article par cette caractérisation. L'étude d'une philosophie politique telle que celle de Rousseau n'a pas un intérêt purement archéologique. En faisant appel au passé d'une pensée philosophique, nous disposons de problèmes et de concepts qui nous obligent à réinterroger le jeu de nos institutions politiques. Une telle réinterrogation est possible à l'aide de ces trois moyens institutionnels qui ont pour effet et pour volonté d'étendre la participation politique des citoyens et de ne pas la réduire à un pouvoir électoral.

Notre intérêt pour la pensée de Rousseau n'est pas seulement archéologique car elle nous permet de définir les lignes d'une philosophie politique critique. Depuis Kant, le terme de critique est associé à l'examen des limites : 
il assigne à la philosophie le rôle d'empêcher la raison d'excéder les limites de ce qui est donné dans l'expérience. La philosophie politique critique a également pour but d'analyser et de surveiller des limites, celles des pouvoirs politiques. Mais, il nous semble que l'on peut déterminer deux types de critique politique à partir de deux définitions de la limite.

Pour la première critique, la conception de la limite se règle sur l'idée que le pouvoir serait par nature excessif et que, par conséquent, il faudrait le limiter en le divisant. La critique est alors une détermination des points limites que chaque pouvoir ne doit pas franchir.

La seconde critique ne refuse pas cette première détermination de la limite, mais elle affirme qu'existe, d'une part, une autre définition de la limite et, d'autre part, un autre enjeu pour la pensée philosophique. Cette définition se construit à partir de l'idée que les institutions politiques tendent à créer une dynamique qui bloque la circulation du pouvoir. Les institutions, au lieu de maintenir des relations de pouvoir mobiles et de permettre aux citoyens d'intervenir pour les modifier, se trouvent bloquées et figées. Ce blocage permet le développement de volontés indépendantes du peuple. Cette définition que nous faisons nôtre, nous permet de mieux déterminer le sens que nous donnons au terme de critique. Élaborer une critique du pouvoir, c'est moins le mettre en accusation en l'assignant à un périmètre de sécurité qu'il ne devrait pas franchir, que de discerner les raisons du blocage de sa mobilité. Ainsi, si, pour la première philosophie critique, la question première est : quelles limites les différents pouvoirs institutionnalisés doivent renoncer à franchir, la question pour notre philosophie critique devient : dans nos institutions politiques, quels sont les mécanismes qui empêchent la circulation du pouvoir et qui limitent l'initiative des citoyens ? L'enjeu critique se modifie car la réflexion sur la limite devient positive : non pas tracer des limites infranchissables pour le pouvoir, mais penser aux conditions de franchir les limites qui empêchent la mobilité du pouvoir et la participation des citoyens $^{110}$.

Il va de soi que cette philosophie critique se refuse à toute prophétie d'une solution historique universelle. Elle se détourne de tous les projets qui prétendent être globaux et radicaux. Elle doit bien plutôt se mettre à l'épreuve de la réalité et de l'actualité propres à chaque pays, à chaque nation. Pour donner un exemple très limité d'une telle critique, on pourrait très succinctement évoquer, pour la France, deux perspectives.

110.Nous rappelons que l'interrogation sur les limites constitutionnelles des différents pouvoirs est tout à fait légitime et nécessaire. La séparation des pouvoirs est une nécessité démocratique. Cela ne fait aucun doute. Mais, l'interrogation sur la limite des pouvoirs ne peut se clore sur cette détermination. La pensée philosophique doit élaborer une conception positive de la limite : l'insérer dans une réflexion des conditions de son franchissement possible pour étendre la mobilité du pouvoir politique. 
La première repose sur ce constat : la mollesse avec laquelle tous les gouvernements de la $V^{\text {ème }}$ République élaborent des lois — et surtout les appliquent - afin d'éviter le cumul des mandats politiques. La possibilité d'un tel cumul est par nature antidémocratique puisque de fait elle favorise la concentration du pouvoir et limite la participation des citoyens au pouvoir. Une démocratie conséquente avec elle-même devrait limiter un mandat électif non seulement dans l'espace politique, mais aussi dans le temps : un député ne devrait ni cumuler plusieurs charges politiques, ni être rééligible un nombre indéfini de fois.

La deuxième perspective pose le problème de la responsabilité politique des gouvernants : comment dans une démocratie, nos gouvernants rendent-ils des comptes ? Cette question est masquée de nos jours par la multiplication des affaires de corruption qui nous oblige à réfléchir en terme de responsabilité pénale des hommes politiques. L'existence d'une telle responsabilité, quand leur fonction dégénère en corruption, ne nous pose aucun problème. Mais ce type de responsabilité n'épuise pas la détermination de cette notion et surtout elle en déplace l'enjeu. Dans une démocratie, doit exister une réelle responsabilité politique des gouvernants qui ne peut pas uniquement se réduire à la sanction de ne pas être réélu. Dans un tel régime, on doit penser à des moyens institutionnels qui rendent responsables les gouvernants. Ces moyens doivent laisser une part active à l'ensemble des citoyens, ils doivent pouvoir intervenir politiquement pour demander des comptes à leur gouvernants. La possibilité d'amender la loi ne devrait pas être du seul ressort de l'Assemblée Nationale et du Sénat, mais aussi, dans une certaine mesure, du ressort du peuple. C'est pour cette raison que la légitimité d'une démocratie se mesure pour Rousseau aux moyens qu'elle met en œuvre pour faire participer le plus grand nombre de citoyens au pouvoir politique, qu'il relève de l'ordre du législatif ou de l'exécutif.

Notre démocratie est-elle animée par la même volonté politique ? 